University of Nebraska - Lincoln

DigitalCommons@University of Nebraska - Lincoln

February 1982

\title{
THE CHROMOSPHERES OF CLASSICAL CEPHEIDS. I. LOW RESOLUTION IUE SPECTRA
}

\author{
Edward G. Schmidt \\ University of Nebraska-Lincoln, eschmidt1@unl.edu \\ Sidney B. Parsons \\ University of Texas at Austin
}

Follow this and additional works at: https://digitalcommons.unl.edu/physicsschmidt

Part of the Physics Commons

Schmidt, Edward G. and Parsons, Sidney B., "THE CHROMOSPHERES OF CLASSICAL CEPHEIDS. I. LOW RESOLUTION IUE SPECTRA" (1982). Edward Schmidt Publications. 24.

https://digitalcommons.unl.edu/physicsschmidt/24

This Article is brought to you for free and open access by the Research Papers in Physics and Astronomy at DigitalCommons@University of Nebraska - Lincoln. It has been accepted for inclusion in Edward Schmidt Publications by an authorized administrator of DigitalCommons@University of Nebraska - Lincoln. 
The Astrophysical Journal Supplement Series, 48:185-198, 1982 February

(C) 1982. The American Astronomical Society. All rights reserved. Printed in U.S.A.

\title{
THE CHROMOSPHERES OF CLASSICAL CEPHEIDS. I. LOW RESOLUTION IUE SPECTRA
}

\author{
EDWARD G. SCHMIDT ${ }^{1}$ \\ Department of Physics and Astronomy, University of Nebraska \\ AND \\ Sidney B. PARSONS ${ }^{1}$ \\ Department of Astronomy, University of Texas at Austin \\ Received 1981 April 22; accepted 1981 July 29
}

\begin{abstract}
We have undertaken a program to study the behavior of chromospheres in classical Cepheids using both ground-based and satellite-based observations. Five stars, $\delta \mathrm{Cep}, \eta \mathrm{Aql}, \beta$ Dor, $\zeta \mathrm{Gem}$, and $l \mathrm{Car}$, have been included, and where possible the various types of data have been obtained simultaneously. The first part of this investigation concerns low dispersion spectra from the IUE satellite. Fine error sensor (FES) magnitudes are presented for the five Cepheids and, in the case of one, $\eta$ Aql, are used to rediscuss the period. The "artificial viscosity dip" predicted by some theoretical calculations may be present in our FES light curve of $\delta$ Cep. The low dispersion spectra have been used to determine continuum magnitudes at various ultraviolet wavelengths, and light curves are shown. The amplitude increases with decreasing wavelength except around $1550 \AA$. Line emission in the short wavelength region (1100 to $1900 \AA$ ) is observed in three of the Cepheids and is found to be phase dependent. Some correspondence is found between the variation of the emission lines and the appearance of bumps on the ultraviolet light curves.
\end{abstract}

Subject headings: stars: Cepheids - stars: chromospheres - ultraviolet: spectra

\section{INTRODUCTION}

The existence of chromospheric activity among cool luminous stars has been known for many years from observations of their $\mathrm{Ca}$ II $\mathrm{H}$ and $\mathrm{K}$ emission cores. Since the advent of balloon-borne spectrometers and space observatories, it has been possible to study this phenomenon further using ultraviolet emission lines. Recently Linsky and Haisch (1979), Bohm-Vitense and Dettmann (1980), and Ayres, Marstad, and Linsky (1981) have used the International Ultraviolet Explorer $(I U E)$ to survey the incidence of chromospheric emission among those stars. Bohm-Vitense and Dettmann showed that chromospheres appear to exist for supergiants cooler than the Cepheid strip, and Linsky and Haisch showed that there is a division between stars exhibiting transition region lines and those which only exhibit chromospheric lines; giants and supergiants cooler than late $\mathrm{G}$ or early $\mathrm{K}$ spectral types do not seem to have transition regions. It also appears that chromospheres do not exist in supergiants earlier than late F.

The chromospheres of Cepheids are of interest both because these stars occupy the region of the H-R diagram between nonvariable stars with chromospheres

\footnotetext{
${ }^{1}$ Guest observer with the International Ultraviolet Explorer Observatory.
}

and those without, and because we might expect that the pulsation will significantly alter the chromosphere. The behavior of $\mathrm{Ca}$ II $\mathrm{H}$ and $\mathrm{K}$ emission cores in Cepheid spectra is well known (Kraft 1957). The emission is transitory and normally appears sometime after minimum light. In stars with periods longer than $\sim 7^{\mathrm{d}}$ the emission is still present at maximum light even though the spectral type is near F5. On the other hand, at minimum light when the spectral types can be as late as $\mathrm{K} 0$, no emission is seen even though nonvariables of that type normally show emission. Schmidt and Weiler (1979) and Parsons (1980) have extended this work to the $\mathrm{Mg}$ II $h$ and $k$ lines in two stars, $\delta$ Cep and $\beta$ Dor. Significant Mg II emission seems to be present throughout the cycle of $\beta$ Dor but has not been seen in $\delta$ Cep.

In order to improve our understanding of Cepheid chromospheres and their time-dependent behavior, we have undertaken a program of observations of chromospheric indicators which includes both ground-based observations of the $\mathrm{Ca}$ II $\mathrm{H}$ and $\mathrm{K}$ lines and observations with IUE of the ultraviolet features. As much as was possible, the ground-based and the satellite observations were made essentially simultaneously. The present paper describes data obtained with the IUE in the low dispersion mode. Later papers in this series will discuss the high dispersion IUE observations of the $\mathrm{Mg}$ II $h$ and 
TABLE 1

MAgNitudes from the Fine ERror SENSOR

\begin{tabular}{|c|c|c|c|c|c|c|c|c|}
\hline $\begin{array}{l}\text { J.D.- } \\
2444000\end{array}$ & Phase & $m$ & $\begin{array}{l}\text { J.D.- } \\
2444000\end{array}$ & Phase & $m$ & $\begin{array}{l}\text { J.D.- } \\
2444000\end{array}$ & Phase & $m$ \\
\hline \multicolumn{9}{|c|}{ a) $\delta$ Cep } \\
\hline $\begin{array}{l}120.85 \\
252.22 \\
252.53 \\
252.77\end{array}$ & $\begin{array}{l}0.251 \\
0.731 \\
0.790 \\
0.834\end{array}$ & $\begin{array}{l}3.95 \\
4.43 \\
4.39 \\
4.24\end{array}$ & $\begin{array}{l}538.46 \\
538.88 \\
540.46 \\
540.97 \\
542.62\end{array}$ & $\begin{array}{l}0.072 \\
0.152 \\
0.445 \\
0.540 \\
0.848\end{array}$ & $\begin{array}{l}3.67 \\
3.80 \\
4.18 \\
4.26 \\
4.23\end{array}$ & $\begin{array}{l}542.65 \\
542.67 \\
542.71 \\
542.86 \\
543.04\end{array}$ & $\begin{array}{l}0.853 \\
0.857 \\
0.864 \\
0.892 \\
0.926\end{array}$ & $\begin{array}{l}4.27 \\
4.21 \\
4.20 \\
4.00 \\
3.78\end{array}$ \\
\hline \multicolumn{9}{|c|}{ b) $n \mathrm{Aql}$} \\
\hline $\begin{array}{l}120.71 \\
207.41 \\
207.50 \\
207.61 \\
207.66\end{array}$ & $\begin{array}{l}0.752 \\
0.833 \\
0.846 \\
0.860 \\
0.868\end{array}$ & $\begin{array}{l}4.38 \\
4.19 \\
4.14 \\
4.12 \\
4.09\end{array}$ & $\begin{array}{l}538.53 \\
538.81 \\
539.04 \\
539.80 \\
540.06\end{array}$ & $\begin{array}{l}0.970 \\
0.008 \\
0.041 \\
0.146 \\
0.183\end{array}$ & $\begin{array}{l}3.68 \\
3.61 \\
3.68 \\
3.82 \\
3.86\end{array}$ & $\begin{array}{l}540.53 \\
540.93 \\
542.79 \\
543.75\end{array}$ & $\begin{array}{l}0.249 \\
0.304 \\
0.564 \\
0.698\end{array}$ & $\begin{array}{l}3.92 \\
3.88 \\
4.31 \\
4.45\end{array}$ \\
\hline
\end{tabular}

c) B Dor

\begin{tabular}{lllllllll}
\hline 120.64 & 0.676 & 3.94 & 252.32 & 0.055 & 3.62 & 540.59 & 0.342 & 4.03 \\
121.12 & 0.725 & 3.88 & 252.67 & 0.090 & 3.65 & 542.91 & 0.579 & 4.19 \\
207.77 & 0.528 & 4.20 & 538.97 & 0.178 & 3.76 & 543.97 & 0.686 & 3.95 \\
207.83 & 0.534 & 4.38 & & & & & & \\
\hline
\end{tabular}

\begin{tabular}{lllllllll}
\hline \multicolumn{7}{c}{ d) $\zeta$ Gem } \\
\hline 120.52 & 0.986 & 3.76 & 538.71 & 0.180 & 3.96 & 542.56 & 0.559 & 4.26 \\
121.02 & 0.035 & 3.78 & 539.98 & 0.305 & 4.13 & 542.97 & 0.600 & 4.22 \\
207.87 & 0.592 & 4.22 & 540.78 & 0.384 & 4.22 & 543.90 & 0.691 & 4.08 \\
\hline \multicolumn{7}{c}{} & \multicolumn{7}{c}{ e) $\ell$ C ar } \\
\hline 120.97 & 0.194 & 3.69 & 539.89 & 0.977 & 3.51 & 542.45 & 0.049 & 3.61 \\
252.43 & 0.891 & 3.66 & 540.71 & 0.000 & 3.49 & 543.82 & 0.087 & 3.60 \\
538.62 & 0.941 & 3.52 & 541.04 & 0.010 & 3.49 & 544.03 & 0.094 & 3.60 \\
\hline
\end{tabular}

$k$ emission cores and the ground-based observations of the $\mathrm{Ca}$ II $\mathrm{H}$ and $\mathrm{K}$ lines.

\section{THE OBSERVATIONS}

\section{a) The Magnitudes from the Fine Error Sensor and the Ephemerides}

Readings from the fine error sensor (FES) on IUE can be converted to magnitudes, $m(\mathrm{FES})$. In Table 1 we list the values obtained during our observations. The phases of four of the stars were calculated using the following ephemerides:

1) $E=(\mathrm{JD}-2436075.485) /\left(5.366270-0.85 \times 10^{-8} E\right)$ for $\delta$ Cep;

2) $E=(\mathrm{JD}-2440905.30) / 9.8426$ for $\beta$ Dor;

3) $E=(\mathrm{JD}-2410639.755) /(10.153517-4.96 \times$ $\left.10^{-7} E\right)$ for $\zeta \mathrm{Gem}$;

4) $E=(\mathrm{JD}-2439031.064) /\left(35.53836+5.06 \times 10^{-5} E\right)$ for $l$ Car.

Parsons (1980) discussed the elements for $\delta$ Cep and $\beta$ Dor using published photometry together with his FES magnitudes. We have adopted his elements. For $\zeta \mathrm{Gem}$, Abt and Levy (1974) and Scarfe (1976) have studied the period, and we have adopted an average of their elements. An examination of our FES magnitudes and the photometry of Moffett and Barnes (1980) shows that these elements are still quite satisfactory. For $l$ Car we have taken the elements from Cogan, Faulkner, and Butler (1980).

Schmidt (1971) studied the period of $\eta$ Aql and found that there seemed to have been a period change about 1961. Since that study, several new sets of photometry results have been obtained, and the interval of time since the period change has increased by a factor of 3 . Therefore, it is worthwhile to discuss the present period of this star. To do this, we have used light curves from the six sources listed in Table 2 . In the previous study of this variable, the dates of maximum were determined by fitting various light curves to a standard curve which came from the data of Mitchell et al. (1964). The more recent light curves of Pel (1976) and Moffett and Barnes (1980) have considerably better phase coverage and smaller scatter so we have fitted all the other data to their curves. However, the fitting to the Mitchell et al. 
TABLE 2

Dates of Maximum for Eta Aquilae

\begin{tabular}{|c|c|c|}
\hline $\begin{array}{l}\text { J.D. } \\
(1)\end{array}$ & $\begin{array}{l}\text { Source } \\
\text { (2) }\end{array}$ & $\begin{array}{c}\text { Observed-Calculated } \\
\text { (3) }\end{array}$ \\
\hline 2437175.36 & Mitchell et al. 1964 & a \\
\hline 39859.47 & Schmidt 1971 & +0.03 \\
\hline 41129.72 & Evans 1976 & -0.02 \\
\hline 41158.43 & Pel 1976 & -0.02 \\
\hline 43691.85 & Moffett and Barnes 1980 & -0.03 \\
\hline 44538.82 & FES magnitudes & +0.08 \\
\hline
\end{tabular}

${ }^{\text {a }}$ Prior to period change.

data has ensured that the phasing of the dates of maximum listed in Table 2 is consistent with those previously determined. For the Schmidt photometry we have redetermined the date of maximum and find it to be 0.09 earlier than he lists. The brightest point of Pel's light curve actually occurs $\sim 0.2$ later than that given in Table 2, while for the Moffett and Barnes photometry, the brightest point coincides with our date of maximum. While the exact time of maximum is uncertain because of gaps in phase coverage, photometric errors, and the fact that the maximum is somewhat flat, the relative phasing from one set of photometry data to another is generally accurate to better than 0.01 of the period. Using the dates of maximum given in the table we have fitted the following new elements for $\eta$ Aql:

$$
E=(\mathrm{JD}-2438000.64) / 7.176843 \text {. }
$$

These are valid after JD $=2438000$ (1962) and confirm the period change suggested previously. The last column of Table 2 lists the differences between the observed dates of maximum and those calculated from equation (1). It can be seen that the derived period fits the observations well.

In Figure 1 the light curves from the FES magnitudes are plotted. Included are the FES magnitudes for these stars given by Parsons (1980). The solid curves in the diagram are smoothed $V$ light curves from Moffett and Barnes which have been shifted by between 0.07 and $0.13 \mathrm{mag}$ to fit the FES magnitudes. The sense of the shift is such that the FES magnitudes are fainter than the $V$ magnitudes. The rms scatter about the smoothed curves for all the stars is $\pm 0.03 \mathrm{mag}$. Thus, as noted by Parsons (1980), the internal accuracy is quite good, and these data can be used in studies of the period behavior of variable stars.

There is an interesting feature in the light curve of $\delta$ Cep at phase 0.85 . One point at that phase falls 0.07 mag below the smoothed light curve and 0.05 mag below the FES readings taken $\sim 1 / 2 \mathrm{hr}$ before and after that phase, suggesting a dip of short duration. Such features have been found in theoretical light curves (see, e.g.,

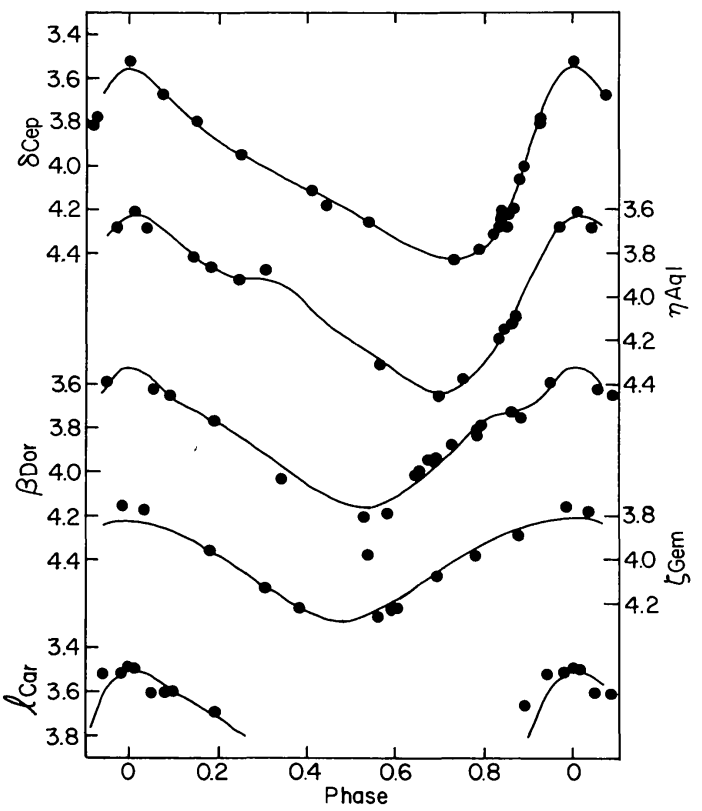

FIG. 1.-Magnitudes from the fine error sensor, $m(\mathrm{FES})$. Data from Table 1 and from Parsons (1980) are plotted. Solid curves are $V$ light curves which have been shifted in zero point to fit the FES magnitudes.

Adams, Castor, and Davis 1980) and are referred to as the "artificial viscosity dip." Additionally, Davis, Moffett, and Barnes (1980) have found such a feature in the $16^{\mathrm{d}}$ Cepheid X Cyg. Although the reality of the dip in the light curve of $\delta$ Cep is somewhat uncertain, it is clearly of sufficient interest that attempts should be made to confirm its presence.

\section{b) The Ultraviolet Observations}

The IUE spectra we have used in this investigation are listed in Tables 3 and 4 . The spectra in Table 3 were obtained with the long wavelength spectrograph and those in Table 4 with the short wavelength spectrograph. In addition to the data which we obtained ourselves with IUE during 1979 and 1980, we have used spectra available from the National Space Science Data Center which were obtained by other investigators. For several of the stars, the combination of our data and that of others has resulted in fairly complete phase coverage. In each table the first column gives the $I U E$ frame number of the exposure, and the following column gives the Julian Date of the midpoint of the exposure or exposures. In many cases exposures were made through both entrance apertures, and columns (4) and (5) give the exposure times. The approximate wavelength range which is well exposed is indicated in the following column. We indicate the observing program in which the data were obtained in the seventh column of each table. The remaining columns are discussed in § III. 


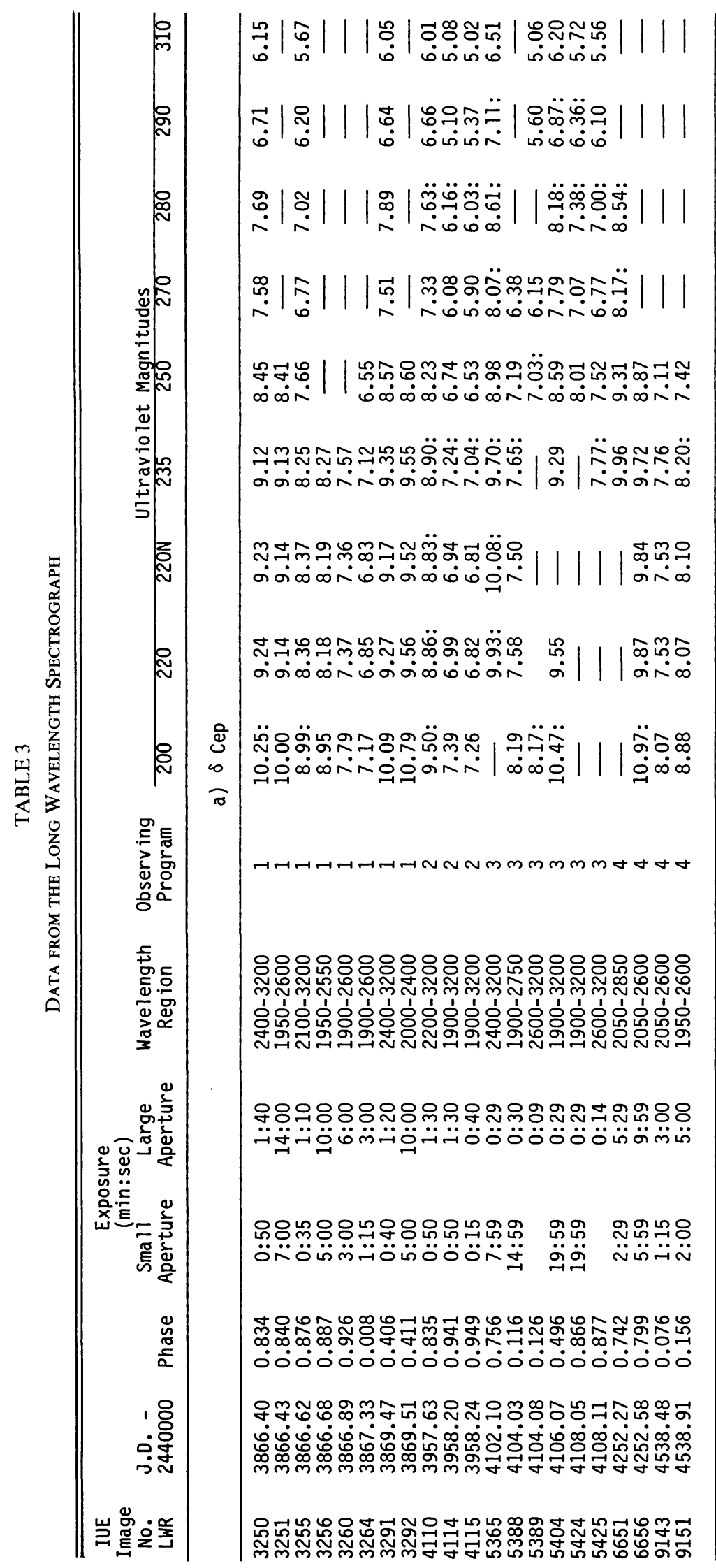

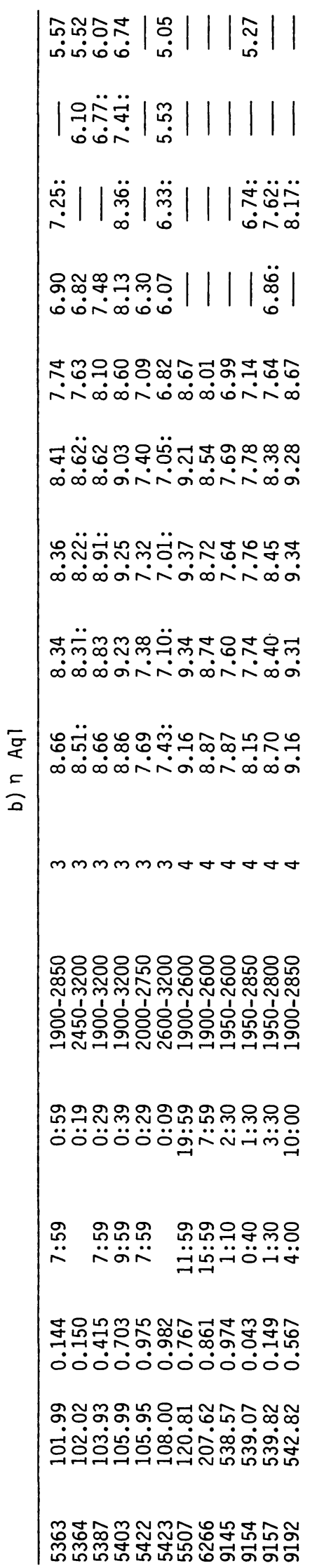




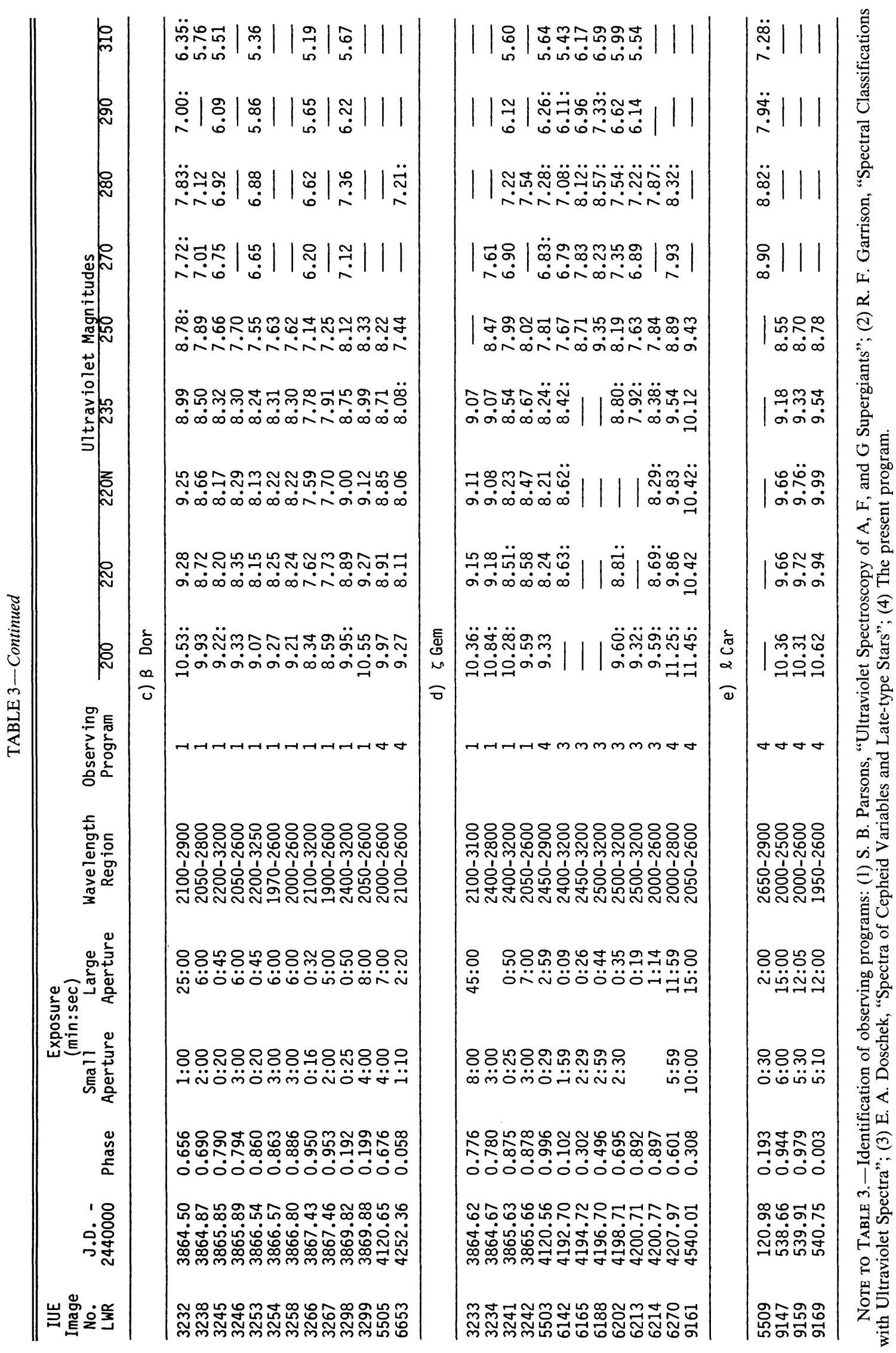




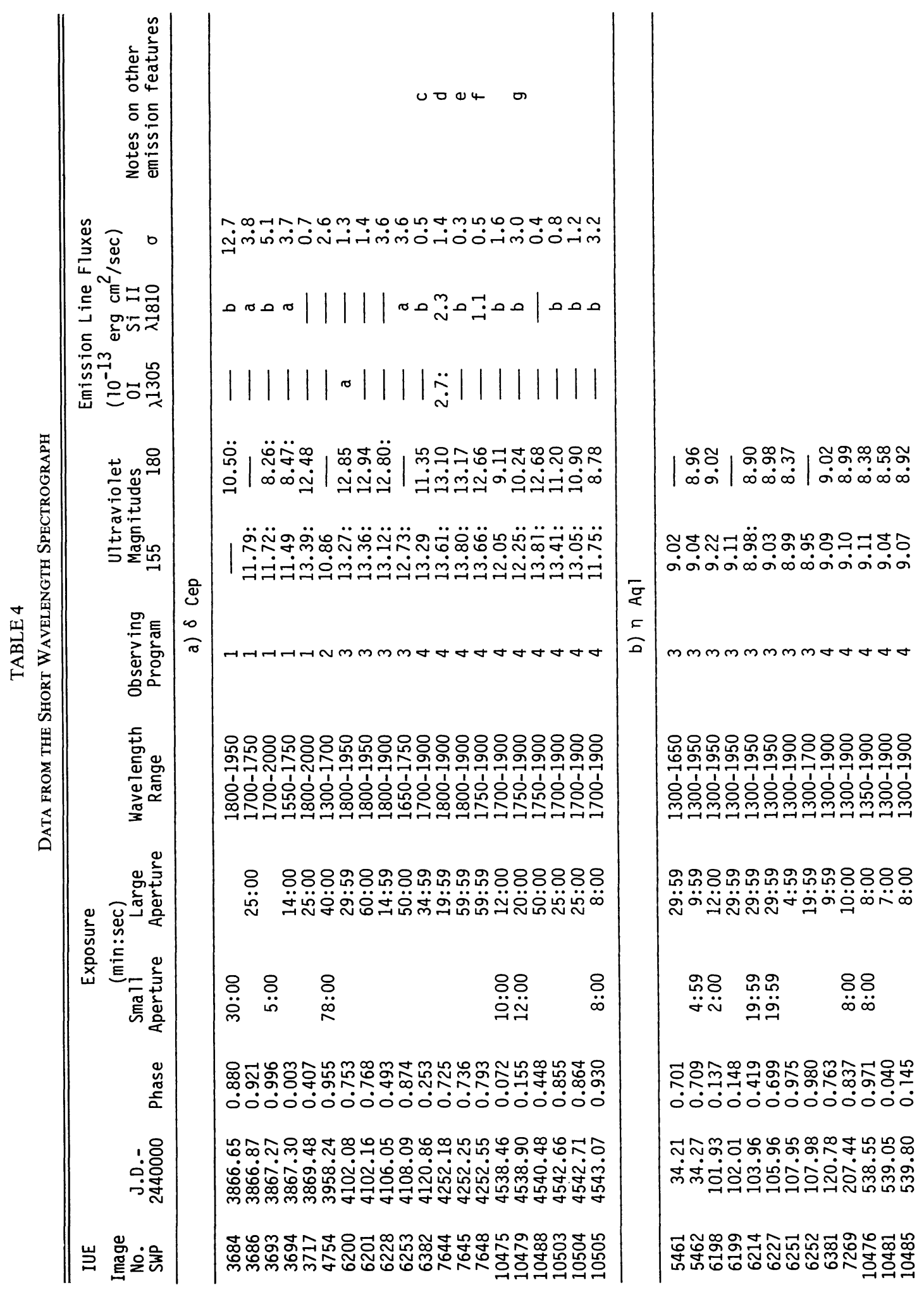




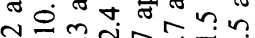
II $\pi=0 \pi$

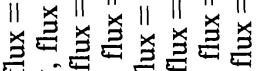

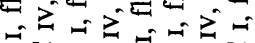

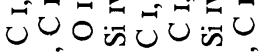

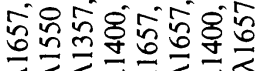

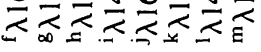

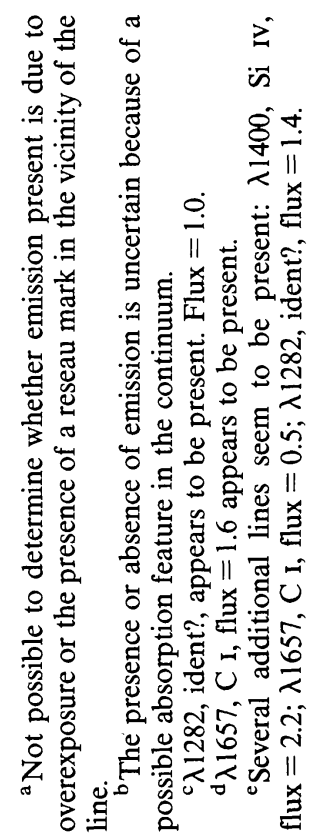




\section{c) The Data Reduction}

Most of the necessary data reduction is carried out by IUE Observatory staff using standard techniques for correcting the images for geometric distortions, transforming the data to intensities, removing background, and extracting the net spectrum in flux numbers. We have transformed the flux numbers to absolute intensity using the calibration of Turnrose, Bohlin, and Harvel (1980) which is now routinely applied to low dispersion data by the IUE staff. There has been some discussion regarding the accuracy of this calibration, but any differences between it and other suggested calibrations are of the order of $10 \%$ or less at the wavelengths of interest here (Bohlin and Holm 1980). This is not important for our interpretation of the data and, of course, does not affect the shapes of the light curves, the variations of line intensities, or comparisons between different stars.

For the short wavelength observations during the first year of operation of $I U E$, the reduction of the data was carried out with an intensity transfer function which contained errors. Of the data we have used, only the Parsons spectra from 1978 are affected. These spectra were corrected with the three agency fourth file method described by Cassatella et al. (1980).

\section{THE CONTINUUM MAGNITUDES}

In order to study the variations of the continuous flux in the ultraviolet, we have calculated the mean fluxes over rectangular passbands at various wavelengths. The wavelengths and the widths of the bands are listed in Table 5. The resulting continuum fluxes are expressed as magnitudes normalized so that zero magnitude corresponds to a flux of $3.64 \times 10^{-9}$ ergs $\mathrm{cm}^{-2} \mathrm{~s}^{-1} \AA^{-1}$ (following Henize et al. 1979). The magnitudes are listed for each frame in Tables 3 and 4.

When exposures were taken through both apertures, we have combined the two to give a weighted average magnitude. The large aperture accepts virtually all the starlight, and the calibration of fluxes refers to it. However, the proportion of light entering the spectrograph through the small aperture varies appreciably from one exposure to another. For this reason, we have scaled the small aperture exposures to match the large aperture exposures before calculating the magnitudes and forming the averages.

Because the quality of the magnitudes depends strongly on the amount of background and the level of the exposure, we have developed a weighting scheme to indicate the quality of the data. The most reliable data should have internal standard deviations of $\sim 0.15 \mathrm{mag}$ for each tabulated value. The data which were judged to have about half-weight should have internal standard deviations about twice as great. Such data are marked with colons in the tables and are plotted as open symbols in the various figures. These internal errors were
TABLE 5

Characteristics of Passbands FOR ULTRAVIOLET MAGNITUDES

\begin{tabular}{|c|c|}
\hline $\begin{array}{c}\text { Central } \\
\text { Wavelength } \\
\text { (§) }\end{array}$ & $\begin{array}{c}\text { Width } \\
(\AA)\end{array}$ \\
\hline 1550 & 150 \\
\hline $1800 \ldots$ & 150 \\
\hline $2000 \ldots$. & 100 \\
\hline $2200 \ldots \ldots$ & 200 \\
\hline $2200 \mathrm{~N} \ldots$ & 100 \\
\hline $2350 \ldots$ & 100 \\
\hline $2500 \ldots$ & 150 \\
\hline $2700 \quad \ldots$ & 100 \\
\hline 2800 & 50 \\
\hline 2900 & 100 \\
\hline 3100 & 100 \\
\hline
\end{tabular}

estimated by comparing pairs of observations which were made at nearly the same phase. Since this includes observations separated by 1 or $2 \mathrm{yr}$ in some instances, the estimated errors should include long-term effects as well as measurement uncertainties. When a spectrum was overexposed, underexposed, or had serious contamination by a fiducial mark within the passband, the magnitude was discarded, and no entry appears in the table.

We have plotted the continuum magnitudes in Figures 2 and 3. In Figure 2 the continuum magnitudes are plotted against the reciprocal wavelengths at selected phases for the various stars. In all the spectra which cover the region, the absorption lines of $\mathrm{Mg}$ II at $\lambda 2800$ $\AA(1 / \lambda=3.57)$ are clearly visible. In the $\lambda 2350$ band $(1 / \lambda=4.25)$ a dip is visible in many of the energy distributions. This is due mainly to a blend of Fe II lines. The slope of the curves between the two shortest wavelengths decreases noticeably at lower temperatures (i.e., near minimum light) as compared with the hotter temperatures. This gives rise to the reduced amplitudes in the $\lambda 1550$ band which are discussed below.

In Figure 3 we have plotted light curves from our continuum magnitudes. The solid lines are scaled $B$ light curves (from Moffett and Barnes 1980) which are included only for comparison. In the case of $\beta$ Dor no scaled $B$ curve is shown because our phase coverage is not adequate to make a meaningful comparison.

During declining light the ultraviolet magnitudes of $\delta$ Cep are fainter and during rising light they are brighter than the B curves. Had we plotted color-color diagrams, this would have manifested itself as looping. The case of $\eta$ Aql is somewhat different, as should be expected from the fact that it is known to have a hot companion (Mariska, Doschek, and Feldman 1980). At $1550 \AA$ the companion dominates the flux, and there is no significant variation, while at $1800 \AA$ the companion apparently dominates except for times near maximum 


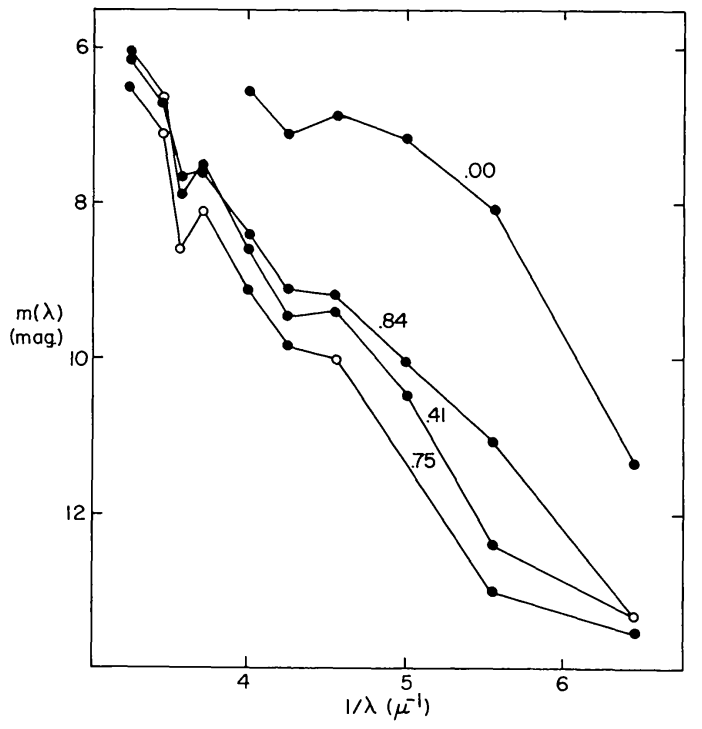

FIG. $2 a$

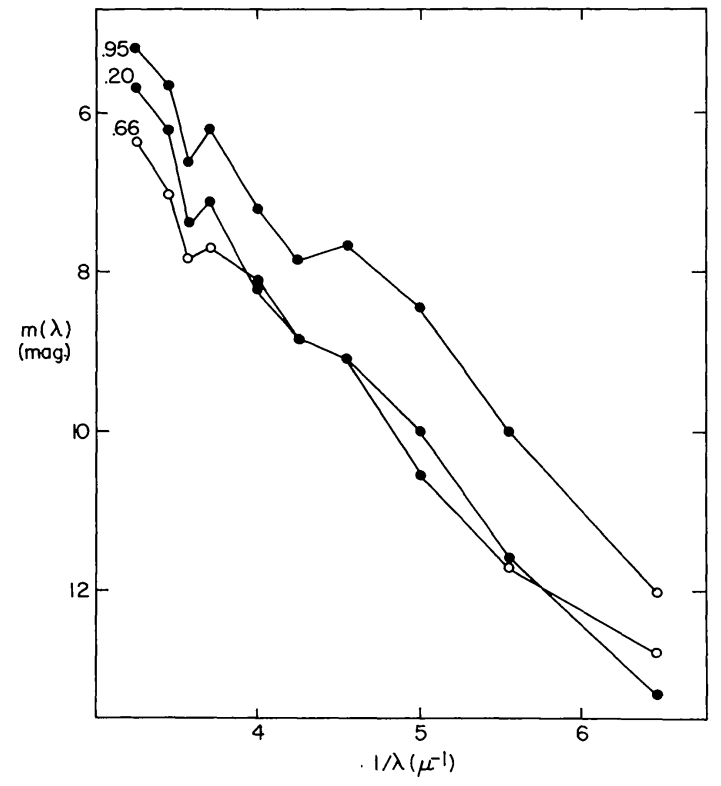

Fig. $2 c$

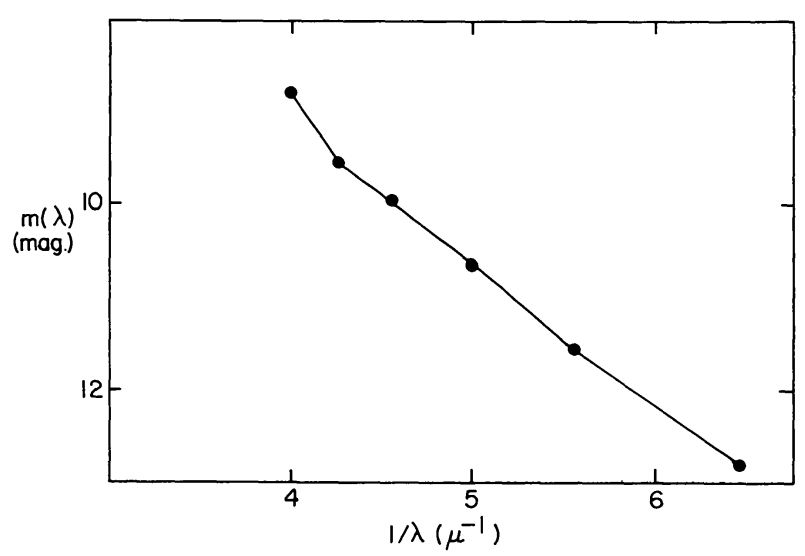

FIG. $2 e$

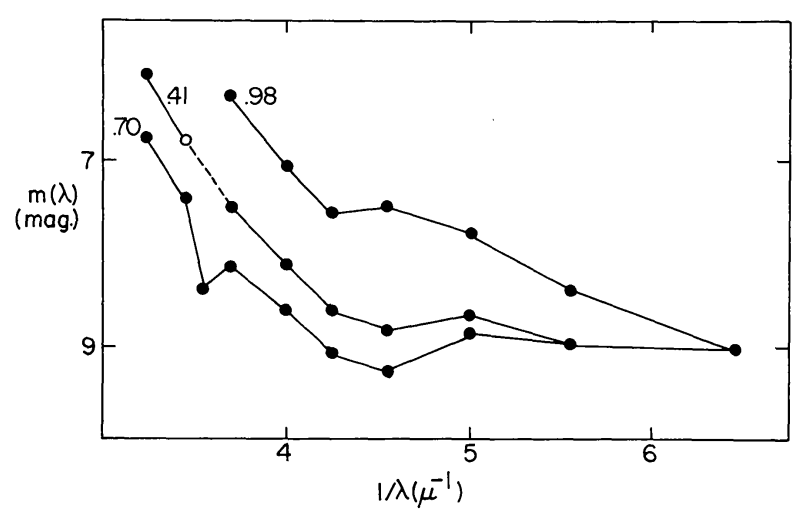

FIG. $2 b$

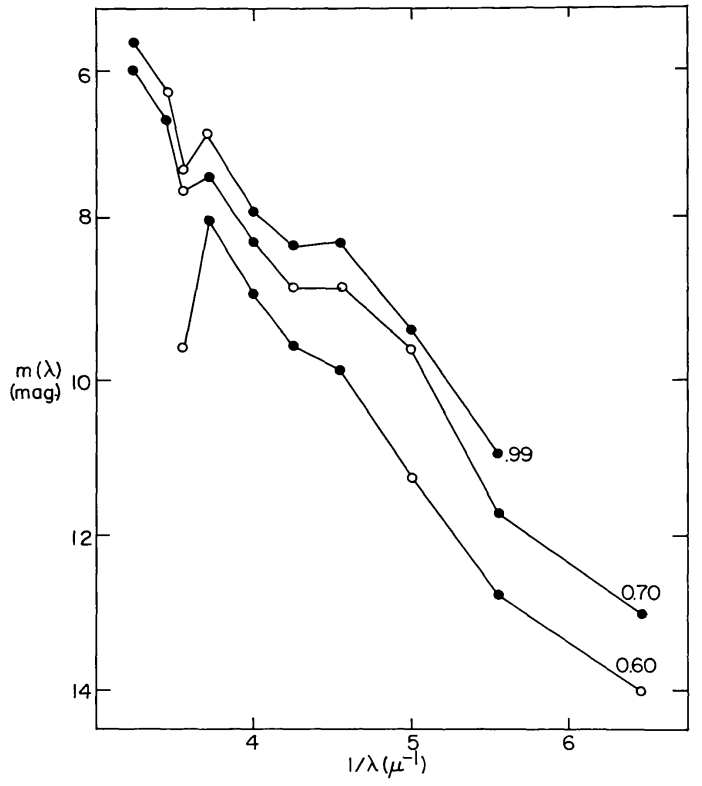

FIG. $2 d$

FIG. 2.-Continuum magnitudes plotted against reciprocal wavelengths for selected phases. Open symbols denote half-weight points (marked by colons in Tables 3 and 4). (a) $\delta$ Cep; (b) $\eta$ Aql; (c) $\beta$ Dor; (d) $\zeta \mathrm{Gem} ;(e) l \mathrm{Car}$. 


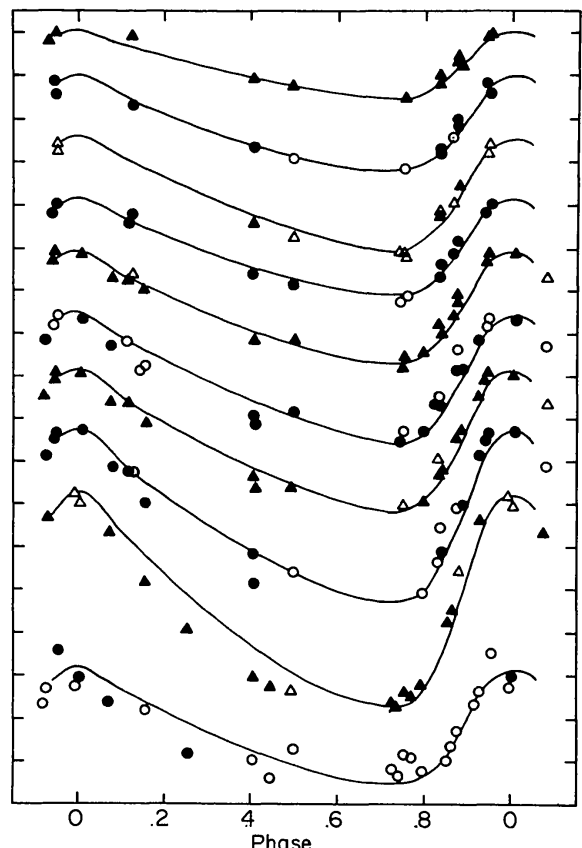

FIG. $3 a$

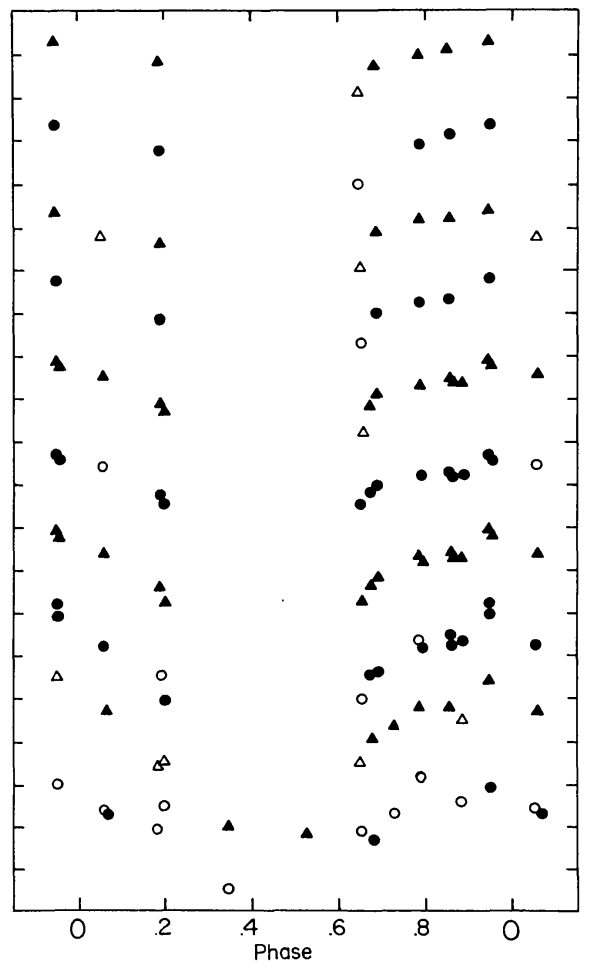

FIG. $3 c$

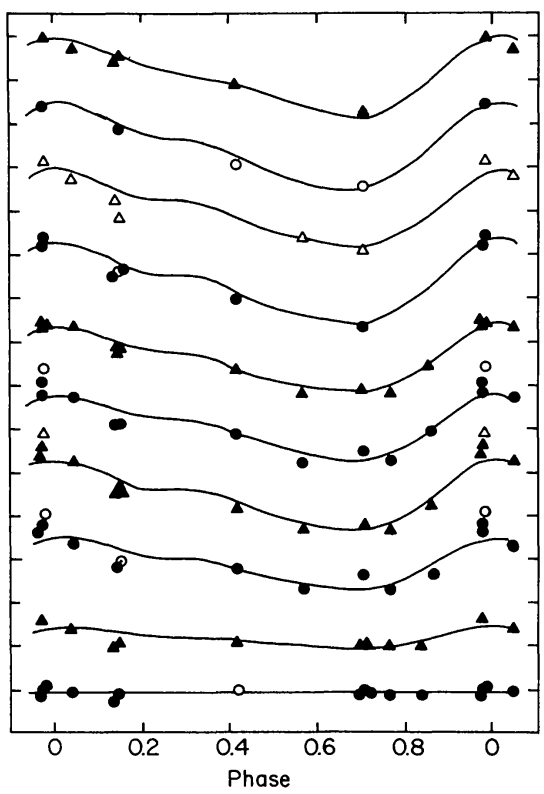

FIG. $3 b$

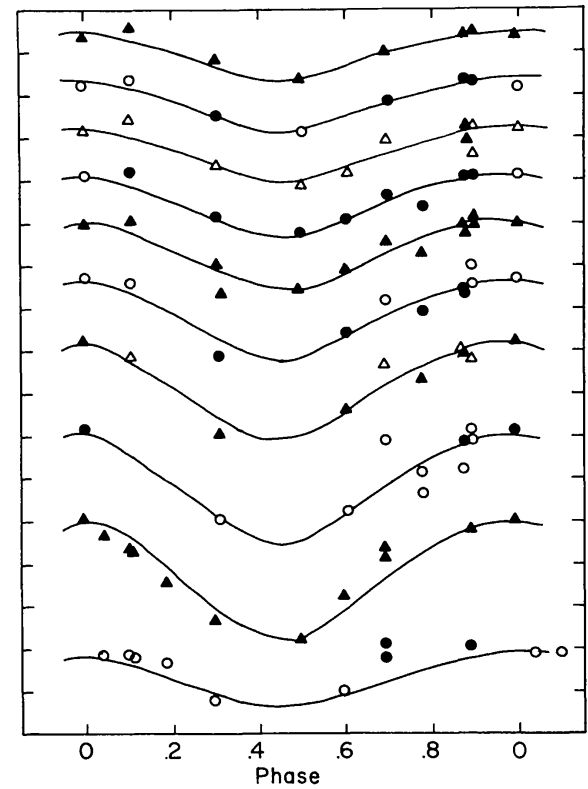

FIG. $3 d$

FIG. 3.-Ultraviolet light curves. Solid curves are scaled $B$ magnitudes. Open symbols denote half-weight points. The longest wavelength curves are at the top, and the symbols are alternated to facilitate identification. Tick marks are 1 mag apart. The curves have been shifted by arbitrary amounts to facilitate plotting. (a) $\delta \mathrm{Cep} ;(b) \eta \mathrm{Aql} ;(c) \beta$ Dor; (d) $\zeta \mathrm{Gem}$. 


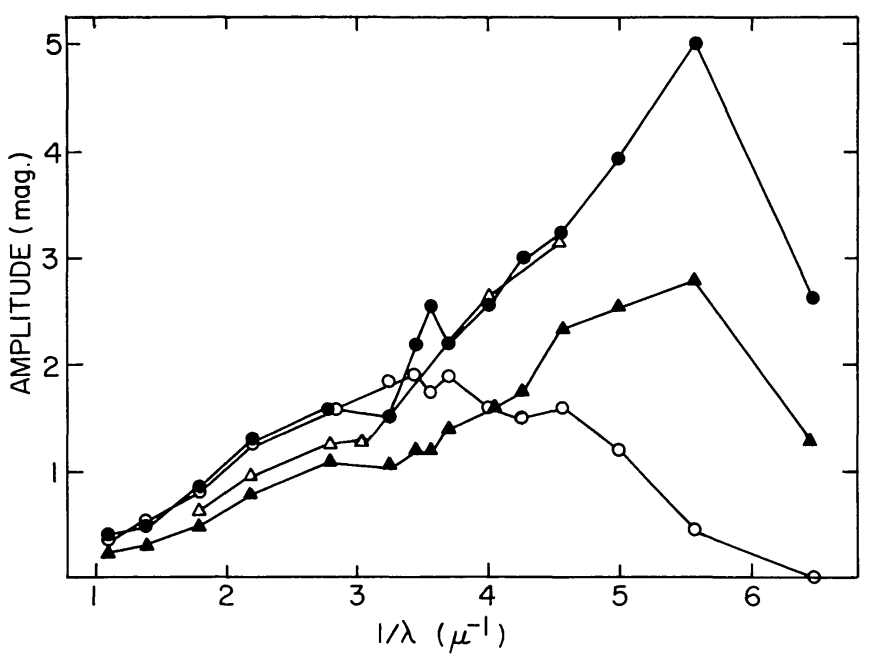

FIG. 4.-Amplitudes plotted against reciprocal wavelength. Stars are indicated by different symbols as follows: solid circles, $\delta$ Cep; open circles, $\eta \mathrm{Aql}$; open triangles, $\beta$ Dor; filled triangles, $\zeta$ Gem.

light. As the wavelength increases, the behavior of the ultraviolet light curves becomes more and more similar to the $B$ curve. In the case of $\zeta \mathrm{Gem}$, we have relatively poor phase coverage of the descending portion of the light curves. However, it appears that the light curves are quite similar in shape to the $B$ curve except around phases 0.7 to 0.8 where a substantial bump appears. Although occasional points scatter by enough to create the bump, it should be noted that three spectra near phase 0.7 all show the bump, and two spectra define the dip at 0.78 . Thus, the feature is likely to be real and is separated from maximum light by a local minimum. Although we do not have sufficient phase coverage to define the form of the curves well for $\beta$ Dor, there is the definite appearance of a shoulder or bump on the curves between phases 0.65 and 0.70 . The data of Hutchinson (1975) and Lub et al. (1979) also show this feature.

Using the fitted $B$ curves as a guide, we have estimated the amplitudes of the three stars for which we have sufficient phase coverage. These are plotted against the reciprocal of the wavelength in Figure 4. For $\beta$ Dor we have plotted the amplitudes from Lub et al. (1979) for wavelengths down to $\lambda 2200$. As has been found for various Cepheids by previous investigators (Hutchinson 1975; Lub et al. 1979), from $1800 \AA$ A longward the amplitude decreases with increasing wavelength. The shortest wavelength amplitudes of $\eta$ Aql deviate from this behavior because of the effects of the companion mentioned above. However, the point at $1550 \AA$ deviates strikingly from this trend for all the stars. It can be seen from the diagram that for $\delta$ Cep the amplitude at $1550 \AA$ is $\sim 3.3$ mag smaller than an extrapolation of the trend at longer wavelengths would suggest, while for $\zeta \mathrm{Gem}$ the discrepancy is $\sim 2.2 \mathrm{mag}$.
The small amplitude at the shortest wavelength is probably due to the presence of the $\mathrm{Si}$ I discontinuity at $1683 \AA$. We have examined the size of this discontinuity in the models of Kurucz (1979). For a gravity of $\log g=$ 2.5 , it increases from $\sim 2 \mathrm{mag}$ at $5500 \mathrm{~K}$ to $4.1 \mathrm{mag}$ at $7000 \mathrm{~K}$. This together with estimated temperature ranges for these stars $(5370$ to $6240 \mathrm{~K}$ for $\delta \mathrm{Cep}$ and 5600 to $6250 \mathrm{~K}$ for $\zeta \mathrm{Gem}$ ), was used to calculate the expected reduction in amplitude caused by the discontinuity. The resulting amplitude reductions, $1.2 \mathrm{mag}$ for $\delta$ Cep and $0.9 \mathrm{mag}$ for $\zeta \mathrm{Gem}$, are less than half what we observed. The use of a lower gravity model, probably more appropriate to these stars, reduces the theoretical amplitude reduction and aggravates the disagreement. Therefore, we conclude that the model atmospheres do not adequately predict the fluxes of these stars in the far ultraviolet.

\section{LINE EMISSION}

The short wavelength exposures were largely obtained to search for emission lines from the chromospheres and transition regions. Some samples of spectra are illustrated in Figure 5. Included are spectra which had some of the strongest lines we have observed and spectra which have no significant emission. For $\eta$ Aql no emission lines can be detected because of flux from the companion.

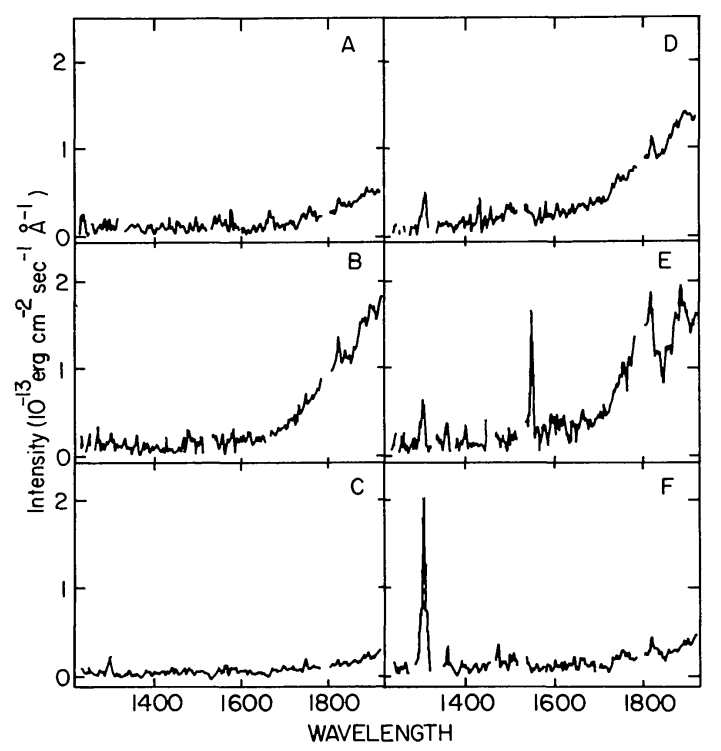

(ค̊)

FIG. 5.-Sample of short wavelength spectra. Panels display spectra for various stars as follows: (a) SWP 7648, $\delta$ Cep; $(b)$ SWP 6380, $\beta$ Dor; (c) SWP 7270, $\beta$ Dor; (d) SWP 7196, $\zeta$ Gem; (e) SWP 10477, $l$ Car; $(f)$ SWP 10513, $l$ Car. Breaks in the spectra are the result of points which are affected by reseau marks. 
Tracings of all the short wavelength spectra were examined to determine what emission lines are present. If an emission feature was above the noise level, was sharp, and had a wavelength corresponding to an expected emission line, it was regarded as real, and the intensity was obtained. In addition, for each spectrum the emission intensity was calculated in several bands known to contain no features. This was used to determine the uncertainties in the line strengths. Table 4 lists the line strengths (in units of $10^{-13} \mathrm{ergs} \mathrm{cm}^{-2} \mathrm{~s}^{-1}$ ) and the standard deviations associated with them. When there is no entry, it signifies that no emission feature was visible, and the standard deviations listed can be used to estimate upper limits on the fluxes. Since the tracings were searched for possible emission lines, the table should contain all features which can be considered significant in the well-exposed spectral range.

The emission line $\lambda 1810 \mathrm{Si}$ II presents special difficulties. As can be seen in the tracings in Figure $5 b, d$, and $e$, there is often a strong absorption line on the longward side of the emission. This makes it difficult to determine whether emission occurs or whether the absorption feature causes the apparent emission. Cases where the emission is narrow and well defined have been accepted as real (e.g., Fig. $5 a, b, d, f$ ), and cases in which the absorption appears to distort or obscure the presence of emission (e.g., Fig. 5e) are so indicated in the table.

It can be seen from Table 4 that $\lambda 1305 \mathrm{O}_{\mathrm{I}}$ is detected in several of the stars. However, $l$ Car is the only star which exhibits other lines consistently. We have plotted the strengths of the emission lines in Figure 6. It can be seen that the various stars differ substantially, as noted by Parsons (1980). In the case of $\delta$ Cep little significant emission has been observed in spite of good phase coverage and a number of quite deep exposures. In $\beta$ Dor the emission of $\lambda 1305 O$ I appears suddenly around phase 0.7 , goes through a maximum $\sim 0.15$ cycles later, and gradually fades during the remainder of the cycle. The only apparently genuine cases of $\lambda 1810$ $\mathrm{Si}$ II emission in that star occur during the time of the $\mathrm{O}$ I maximum. In $\zeta$ Gem we have two spectra at nearly the identical phase of 0.69 , and there is a significant difference in the $\lambda 1305$ emission. It is possible that the turnon of the emission occurs at about this point in the cycle and that it is not exactly the same in different cycles. There appears to be less variation in the $\lambda 1305$ emission during the cycle of this star than was the case in $\beta$ Dor. In fact, we found no phase for which the emission was not present in $\zeta$ Gem. It also seems to present more activity in the $\lambda 1810$ line, although difficulties in detecting this line make any firm conclusion difficult. Finally, looking at the data for $l$ Car, we see that during the time we observed this star, near maximum light, there is emission in the $\lambda 1305$ line, in

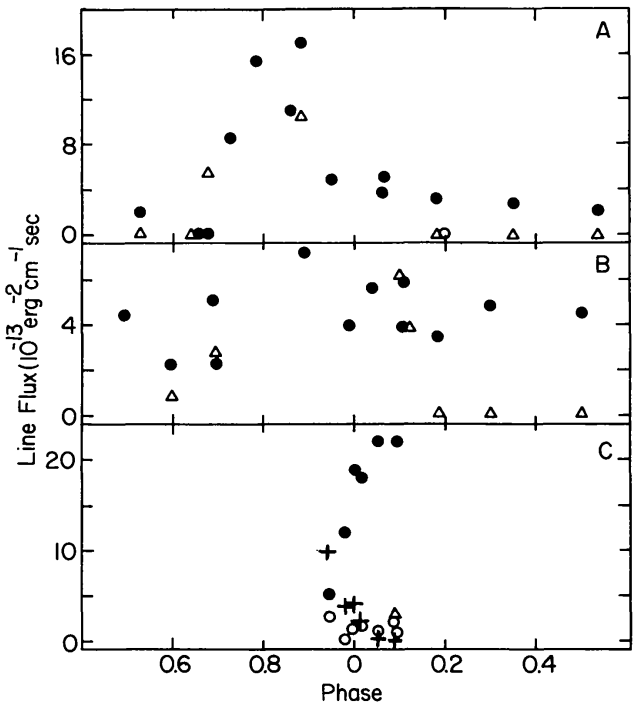

FIG. 6.-Variations of emission line strengths with phase. Symbols denote lines as follows: solid circles, $\lambda 1305 \mathrm{O}$ I; open circles, $\lambda 1355 \mathrm{O}$ I; crosses, $\lambda 1550 \mathrm{C}$ IV; open triangles, $\lambda 1810 \mathrm{Si}$ II. $(a)$ $\beta$ Dor; (b) $\zeta \mathrm{Gem}$; (c) $l$ Car.

$\lambda 1355 \mathrm{O}$ I, $\lambda 1550 \mathrm{C}$ IV, and $\lambda 1810 \mathrm{Si}$ II. During this interval around maximum light, it can be seen that $\lambda 1305$ increases in strength and all the other lines fade.

Ayres, Marstad, and Linsky (1981) have surveyed the spectra of late-type stars in the short wavelength region. We can use their data to compare our line strengths for Cepheids with nonvariable stars. The data for this comparison are contained in Table 6 . The second column gives the mean bolometric flux at the Earth corrected for interstellar extinction. The bolometric corrections come from Sandage and Gratton (1963) and are consistent with the scale of Johnson (1966). The third column gives the reddening taken from Dean, Warren, and Cousins (1978). The mean fluxes of the emission lines were obtained by averaging over the cycles when the line was detected. In order to estimate the upper limit on the line strength when it was not detected, we formed average spectra for each star from the least noisy of the individual spectra listed in Table 4. The average spectra were then used to estimate upper limits (at the 2 $\sigma$ level) of the line strengths. These upper limits are very similar to what would be obtained by combining the upper limits inferred from the errors in Table 4. For those cases when the lines were detected we also list the maximum strengths normalized by the bolometric flux.

In Figure 7 we compare the Cepheids with the nonvariables from the list of Ayres, Marstad, and Linsky (1981). We have corrected their data for interstellar extinction in the same way we did for the Cepheids. In the case of the $\lambda 1305 \mathrm{O}$ I emission, there is a wide range in strength among the nonvariable stars at a given color. The Cepheids generally fall below most of the super- 
TABLE 6

Mean Stellar Parameters

\begin{tabular}{|c|c|c|c|c|c|c|c|c|c|}
\hline \multirow[b]{2}{*}{ STAR } & \multirow{2}{*}{$\begin{array}{c}\left\langle f_{\mathrm{bol}}\right\rangle_{0} \\
\left(10^{-7} \mathrm{ergs} \mathrm{cm}^{-2} \mathrm{~s}^{-1}\right)\end{array}$} & \multirow[b]{2}{*}{$E(B-V)$} & \multirow[b]{2}{*}{$\langle\boldsymbol{B}\rangle_{0}-\langle V\rangle_{0}$} & \multicolumn{2}{|c|}{$\begin{array}{c}\left\langle f_{\text {line }}\right\rangle \\
\left(10^{-13} \mathrm{ergs} \mathrm{cm}^{-2} \mathrm{~s}^{-1}\right)\end{array}$} & \multicolumn{2}{|c|}{$\begin{array}{c}\left\langle f_{\text {line }}\right\rangle_{0} /\left\langle f_{\text {bol }}\right\rangle_{0} \\
\left(10^{-7}\right)\end{array}$} & \multicolumn{2}{|c|}{$\begin{array}{r}f_{\text {line }, 0} /\left\langle f_{\text {bol }}\right\rangle_{0}\left(10^{-7}\right) \\
\text { AT MAXIMUM StRENGTH }\end{array}$} \\
\hline & & & & $\lambda 1305$ & $\lambda 1550$ & $\lambda 1305$ & $\lambda 1550$ & $\lambda 1305$ & $\lambda 1550$ \\
\hline$\delta$ Сep $\ldots$ & 8.6 & 0.09 & 0.57 & $<1.7$ & $<1.7$ & $<4.3$ & $<3.9$ & & $\ldots$ \\
\hline$\beta$ Dor .. & 10.7 & 0.09 & 0.71 & 5.1 & $<2.3$ & 10. & $<4.2$ & 34. & $\ldots$ \\
\hline$\zeta$ Gem... & 8.1 & 0.03 & 0.79 & 4.6 & $<1.2$ & 7.3 & $<1.8$ & 12. & $\cdots$ \\
\hline$l$ Car ... & 15.5 & 0.17 & 1.11 & $\ldots$ & $\ldots$ & $\ldots$ & $\ldots$ & 60. & 23. \\
\hline
\end{tabular}

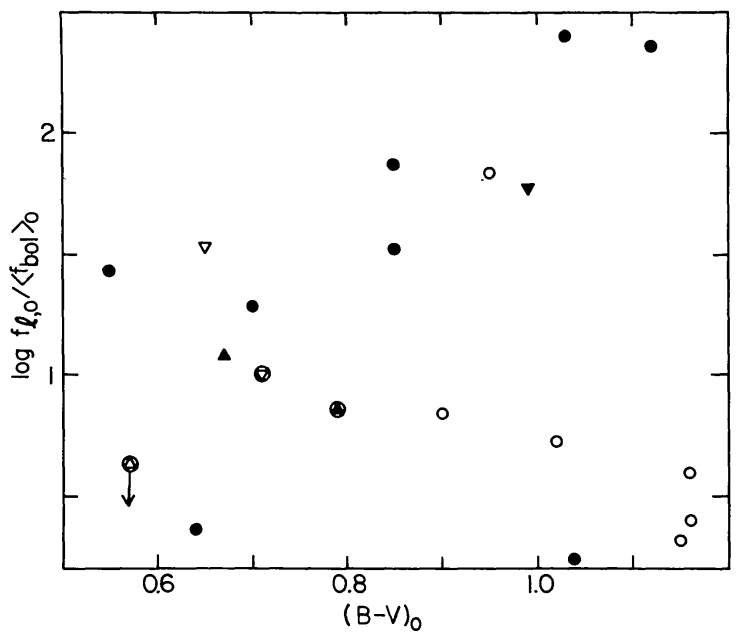

FIG. $7 a$

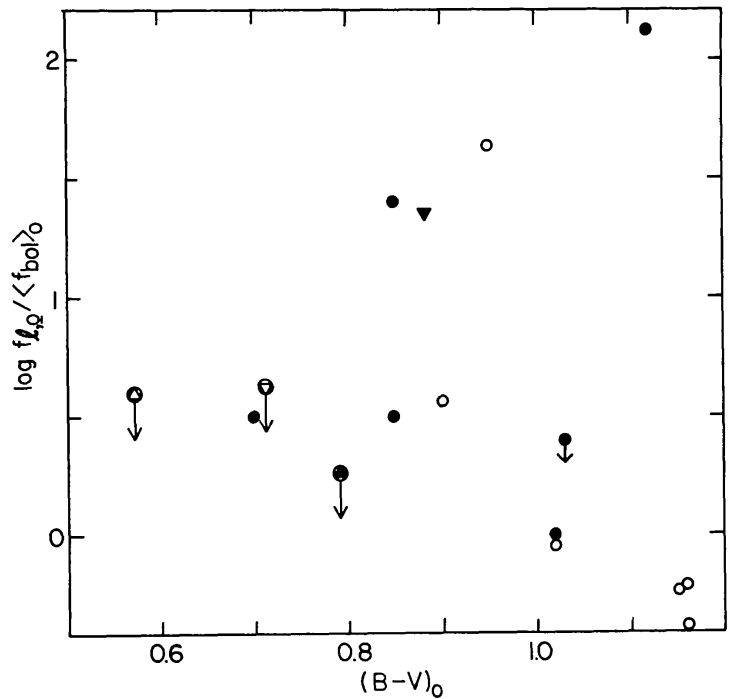

FIG. $7 b$

FIG. 7.-Radiative loss rates in $\lambda 1305 \mathrm{O}$ I and $\lambda 1550 \mathrm{C}$ IV. Filled circles-luminosity class I and II nonvariable stars; open circles - luminosity class III nonvariable stars; open triangles with vertex up $-\delta$ Cep; open triangles with vertex down $-\beta$ Dor; filled triangles with vertex up - $\zeta$ Gem; filled triangles with vertex down $-l$ Car. Circled symbols for the Cepheids denote means over the cycle. Arrows indicate that some of the points are upper limits. Panel $(a)$ shows the data for $\lambda 1305 \mathrm{O}$ I, and panel $(b)$ the data for $\lambda 1550 \mathrm{C}$ IV.

giants but are comparable at the phases of strongest emission. Because the sample of nonvariables is small, this should be regarded with caution. In addition, some of the nonvariables have hybrid spectra (Hartmann, Dupree, and Raymond 1980) and it is possible that the chromospheres of such stars vary in time (Stencel et al. 1980). Thus, a larger sample of nonvariables is needed to clarify the situation.

We have only detected the $\lambda 1550 \mathrm{C}$ IV line in one of the stars in our sample. Hence, in Figure $7 b$ there are only upper limits for three of the stars. It can be seen that both these upper limits and the one point for $l \mathrm{Car}$ are comparable with the nonvariable stars at similar color. Thus, we conclude from this that most of our exposures were not deep enough to detect the presence of transition regions in any stars except $l$ Car. Clearly a project for the future would be to obtain deeper exposures of some of these stars and determine whether they possess transition region lines.
There may be a connection between the appearance of emission lines and the appearance of the ultraviolet light curves. Both $\zeta$ Gem and $\beta$ Dor show emission lines and have bumps on the rising branches of their ultraviolet light curves, while $\delta$ Cep lacks both features. Furthermore, the emission lines strengthen at the phase of the continuum bumps, or slightly after. This would seem to suggest that the disturbance which gives rise to the bumps also excites the chromosphere. In a later paper, in which the $\mathrm{Mg}$ II and Ca II emission lines will be analyzed, we will discuss this question further.

We are indebted to the staff of the $I U E$ Observatory for their help in obtaining and reducing these observations. Discussions with Dr. E. J. Weiler at the early stages of this work are also gratefully acknowledged. This investigation has been supported by the National Aeronautics and Space Administration under grants NSG 5328 and NSG 5391. 


\section{REFERENCES}

Abt, H. A. and Levy, S. G. 1974, Ap. J (Letters), 188, L75.

Adams, T. F., Castor, J. I., and Davis, C. G. 1980, in Current Problems in Stellar Pulsation Instabilities, ed. D. Fischel, J. R. Lesh, and W. M. Sparks (NASA Tech. Memo. 80625), p. 175.

Ayres, T. R., Marstad, N. C., and Linsky, J. L. 1981, preprint.

Bohlin, R., and Holm, A. 1980, NASA IUE Newsletter, 10, 37.

Bohm-Vitense, E., and Dettmann, T. 1980, Ap. J., 236, 560.

Cassatella, A., Holm, A., Ponz, D., and Schiffer, F. H. 1980, NASA IUE Newsletter, 8, 1 .

Cogan, B. C., Faulkner, D. J., and Butler, S. J. 1980, Astr. Ap., 86, 283.

Davis, C. G., Moffett, T. J., and Barnes, T. G. 1981, Ap. J., 246, 914.

Dean, J. F., Warren, P. R., and Cousins, A. W. J. 1978, M.N.R.A.S., 183, 569

Evans, N. R. 1976, Ap. J. Suppl., 32, 399.

Hartmann, L., Dupree, A. K., and Raymond, J. C. 1980, Ap. J. (Letters), 236, L143.

Henize, K. G., Wray, J. D., Parsons, S. B., and Benedict, G. F. 1979, Catalog of Far-Ultraviolet Objective-Prism Spectrophotometry: Skylab Experiment S-019, Ultraviolet Stellar Astronomy (NASA Ref. Pub. 1031).

Hutchinson, J. L. 1975, in Cepheid Modeling, ed. D. Fischel and W. M. Sparks (NASA SP-383), p. 5.
Johnson, H. L. 1966, Ann. Rev. Astr. Ap., 4, 193.

Kraft, R. P. 1957, Ap. J., 125, 336.

Kurucz, R. L. 1979, Ap. J. Suppl., 40, 1.

Linsky, J. L. 1980, Ann. Rev. Astr. Ap., 18, 439.

Linsky, J. L., and Haisch, B. M. 1979, Ap. J. (Letters), 229, L27.

Lub, J., van Paradijs, J., Pel, J. W., and Wesselius, P. R. 1979, Astr. Ap., 72, 82 .

Mariska, J. R., Doschek, G. A., and Feldman, U. 1980, Ap. J. (Letters), 238, L87.

Mitchell, R. I., Iriarte, B., Steinmetz, D., and Johnson, H. L. 1964 Bol. Obs. Tonantzintla y Tacubaya, 3, 153.

Moffett, T. J., and Barnes, T. G. 1980, Ap. J. Suppl., 44, 427.

Parsons, S. B. $1980, A p . J ., 239,555$.

Pel, J. W. 1976, Astr. Ap. Suppl., 24, 413.

Sandage, A., and Gratton, L. 1963, in Star Evolution ed. L. Gratton (New York: Academic), p. 11.

Savage, B. D., and Mathis, J. S. 1979, Ann. Rev. Astr. Ap., 17, 73. Scarfe, C. D. 1976, Ap. J., 209, 141.

Schmidt, E. G. 1971, Ap. J., 165, 335.

Schmidt, E. G., and Weiler, E. S. 1979, A.J., 84, 231.

Stencel, R. E., Mullan, D. J., Linsky, J. L., Basri, G. S., and Worden, S. P. 1980, Ap. J. Suppl., 44, 383.

Turnrose, B., Bohlin, R., and Harvel, C. 1980, NASA IUE Newsletter, 8, 28.

S. B. PARSONS: Department of Astronomy, University of Texas, RLM 15.212, Austin, TX 78712

E. G. SchmidT: Department of Physics and Astronomy, University of Nebraska, Lincoln, NE 68588 